\title{
Traffic analysis and network bandwidth provisioning tools for academic information networks
}

\author{
Shunji Abe1, Toru Hasegawa ${ }^{2}$, Shoichiro Asano ${ }^{3}$ \\ ${ }^{1,3}$ National Institute of Informatics \\ ${ }^{2} K D D I R \& D$ Laboratories Inc. \\ ${ }^{1}$ The Graduate University for Advanced Studies (SOKENDAI)
}

\begin{abstract}
A set of traffic analysis and network bandwidth provisioning tools for support of the Science Information Network (SINET) is presented. The software structure and functionality are also described. The tools provide an estimation of tail probability based on an approximation using Fractional Brownian Motion (FBM) tail probability, and the tools also enable calculation of the average queueing buffer waiting time using the MMPP/G/1 waiting time approximation method. The estimations provided by these tools are consistent with simulations based on observed traffic data.
\end{abstract}

\section{KEYWORDS}

traffic analysis, network provisioning, self-similar traffic, long-range dependent traffic, FBM traffic, MMPP

\section{Introduction}

Internet traffic is characterized as having a self-similar or long-range dependent properties, and these properties are known to affect the quality of service (QoS) of communications [1],[2] due to longer queueing delays and packet loss than in Poisson traffic. Traffic on the Science Information Network (SINET) has also been shown to have a long-range dependent property [3],[4], necessitating network bandwidth provisioning and traffic controls that take this long-range dependence into consideration.

To support the creation of these types of networks, our group has developed traffic analysis and network bandwidth provisioning software tools for the Linux operating system. These tools allow analysis of the packet traffic observed using a traffic capturing tool such as the tcpdump software[6]. The Hurst parameter $(H)$ and queueing buffer tail probability can then be estimated from this analysis. The Hurst parameter is

Received November 11, 2004; Revised February 14, 2005; Accepted February $15,2005$.

\{1)abe@nii.ac.jp, 2)hasegawa@kddilabs.jp, 3)asano\}@nii.ac.jp the main parameter in self-similar or long-range dependent traffic analysis, and the tail probability is an important value required to estimate the buffer overflow probability in network bandwidth provisioning.

This paper presents the structure of this software and the functions of the tools. The estimation function for the tail probability is realized using the approximation method of Fractional Brownian Motion (FBM) tail probability, and the average queueing buffer waiting time is determined using the waiting time approximation method MMPP/G/1. Estimations by these two methods are compared with simulations based on observed traffic data to demonstrate the effectiveness of the proposed tools.

\section{Software construction and Tool functionality}

The tools were implemented using Java-based graphical user interfaces (GUIs), and all operations were executed via these GUIs. The GUIs have a hierarchical structure, as shown in Fig. 1, with the functions termed Extraction, Analyzer, Plot, and User Defined Program. The Analyzer GUI consists of 17 functions for traffic analysis. The actual GUI displays 


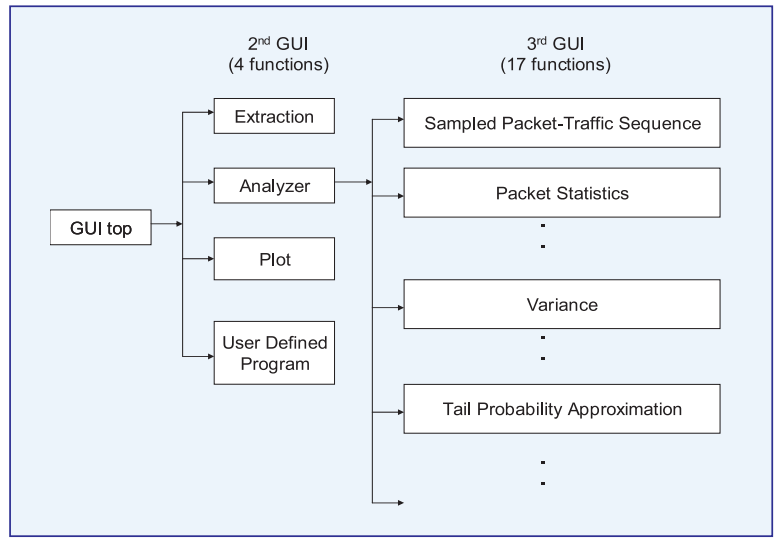

Fig. 1 Hierarchical GUI structure

are shown in Figs. 2 and 3. Figures 2(a) and 3(a) both show the main screen.

\subsection{Extraction function}

The processing flow for the Extraction function (Fig. 2(b)) is shown in Fig. 4. The Extraction function extracts the packet arrival time and packet length from Libpcap-based data captured by programs such as tcpdump. Libpcap [6] is a common program used to capture the packet stream. The extracted data is stored as a time sequence, and the start and end times for extraction can be specified.

\subsection{Analyzer functions}

As shown in Fig. 2(c), the Analyzer function encompasses a total of 16 functions (excluding Delete). These functions process the packet stream data extracted by the Extraction function and output the results for estimation of the Hurst parameter. These functions can also be used to approximate tail probability and average waiting time for a router queueing buffer.

The Hurst Parameter is estimated using the Autocorrelation, Variance, or R/S Analysis functions. These functions sample the extracted data in intervals of $\tau$ and calculate the statistics as a function of discrete time with base $\tau$. The Hurst Parameter is then estimated from the graphed statistics through further manual calculations. The Analyzer functions also include a function to display the results graphically, enabling the creation of user-defined operations, such as addition of a graph.

The tail probability is calculated using the Tail Prob. Approx. function from the results of either the Variance or Relaxation time functions. The result used depends on the approximation method employed to obtain tail probability, as explained below. The FBM model is used for this approximation, requiring approximation of the sampled packet stream by a Gaussian process. The K-S test function, based on the well-known Kolmogorov-Smirnov test [17], is used to check for this packet stream property.

Two different methods for calculating the average waiting time are provided by the program: the Markov Modulated Poisson Process (MMPP) and the Queueing Network Analyzer (QNA). The MMPP method uses the results of the Variance, IDC (index dispersion for counts), and 3rd Central Moment functions. In the QNA method, the results of the IDC and the Relaxation Time functions are used. As the details

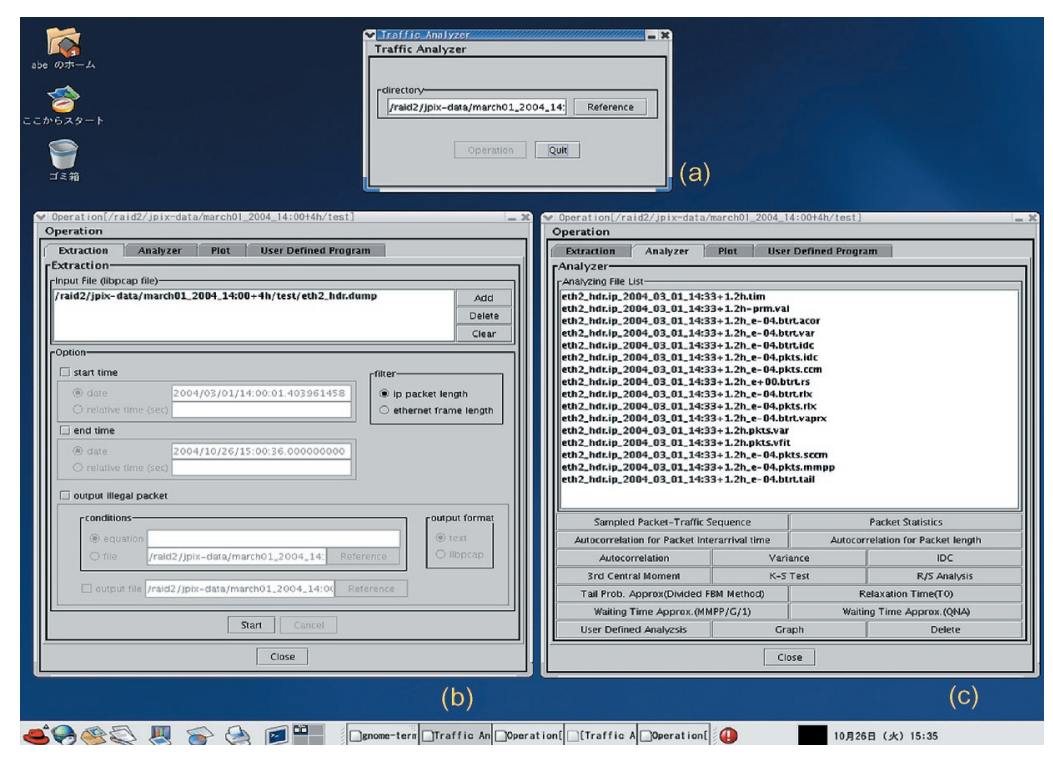

Fig. 2 GUI display 


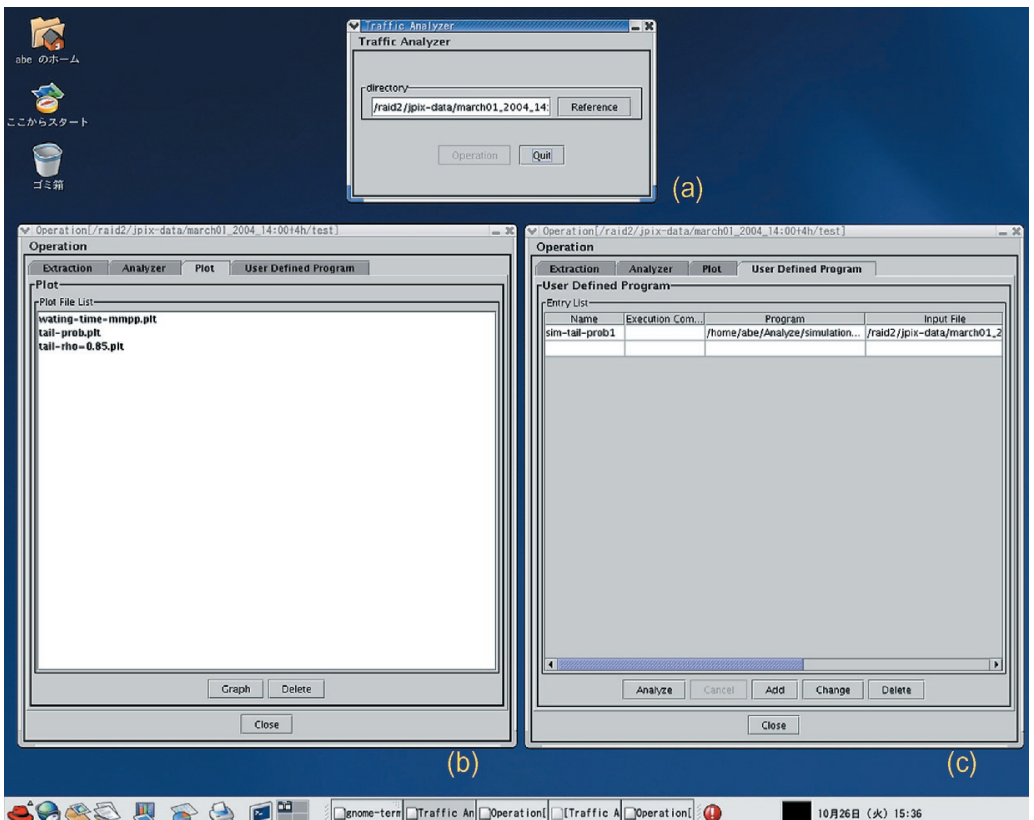

Fig. 3 GUI display

of the QNA method have been reported previously [5], only the MMPP method is described in this paper.

The Sampled Packet-traffic Sequence function can also be used to produce the $\tau$-sample packet sequence data form the extracted data. However, the Autocorrelation, Variance, and R/S Analysis functions already include this function.

In the User Defined Analysis function, parameters required for certain functions can be registered before execution as parameter sets for custom operations. The remaining functions, - Packet Statistics, Autocorrelation for Packet Inter-arrival Time, and Autocorrelation for Packet Length - provide basic statistics, such as the average, variance, and autocorrelation characteristics for the packet inter-arrival time and packet length.

\subsection{Plot function}

This function graphically displays the results obtained using the Analyzer functions in Fig. 3(b). This function is duplicated as an Analyzer function, but in this case allows the graphical output to be reshaped and saved as a postscript file.

\subsection{User Defined Program function}

Any program developed by the user can be executed via this function (Fig. 3(c)). This is very convenient for users requiring functions that are not provided among the Analyzer functions. This function also enables execution of traffic simulation programs using real network traffic data. Simulation results can be compared with estimated tail probability and average waiting time to evaluate the accuracy of the estimations. This function is very simple to use. The userdefined program is registered in the Program column, and the input data file, parameter file, and output file are associated with the program. For user-defined programs written in Linux shell script, the information is input as follows:

\section{\#!/bin/bash}

inputfile $=\$ 1$

parameter $=\$ 2$

outputfile $=\$ 3$

command $=$ user_pro

exec \$command \$inputfile \$parameter \$outputfile

In other cases, shell commands such as /bin/bash must be registered in the Execution Command column.

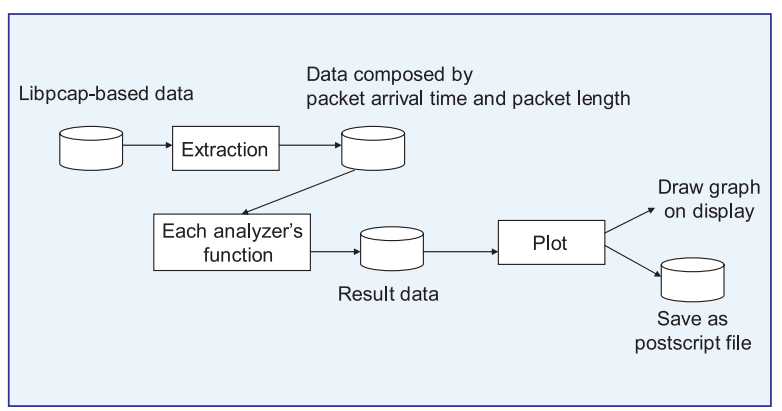

Fig. 4 Processing flow 


\section{Traffic analyzer tools}

The traffic analyzer tools are used to estimate the Hurst parameter for long-range dependent traffic, and to calculate FBM tail probability and MMPP average waiting time for a queueing buffer.

\subsection{Long-range dependence}

The definition of long-range dependence is as follows. Let $X_{n}(n=1,2, \ldots)$ be a sampled process, and be a discrete time with the unit time of $\tau$. Assuming that the sample process $X_{n}$ is a weakly stationary process (WSP), the autocorrelation function $r(k)=\mathrm{E}\left[\left(X_{n}-\right.\right.$ $\left.\mu)\left(X_{n+k}-\mu\right)\right] / \sigma^{2}$, where $\mu$ is the average and $\sigma^{2}$ is the variance of $X_{n}$, is independent of $n$. The sampled process has long-range dependence when the autocorrelation function satisfies the condition

$$
r(k) \approx L(k) k^{-\beta} ; k \rightarrow \infty,
$$

where $L(k)$ is a slowly varying function satisfying $\lim _{k \rightarrow \infty} L(x k) / L(k)=1$ for any $x>0$, and $\beta$ is a decay factor satisfying $0<\beta<1$ for long-range dependence. Such functions can be replaced with a constant function such as $\log (k)$.

Let a random process $S_{n, m}$ be defined as the sum of $m$ consecutive numbers from $X_{n}$, that is,

$$
S_{n, m} \equiv \sum_{j=(n-1)}^{n m} X_{j+1},
$$

then, we also define

$X_{n}^{(m)} \equiv \frac{S_{n, m}}{m}$.

When $X_{n}$ has long-range dependence and $L(k)$ is a constant, the variance-time function $\operatorname{Var}\left[X_{n}^{(m)}\right]$ can be derived using Theorem 2.2 of reference [7] as

$$
\operatorname{Var}\left[X_{n}^{(m)}\right] \approx c m^{-\beta} ; m \rightarrow \infty,
$$

where $c$ is a constant and $0<\beta<1$. Moreover, $\beta$ can be expressed as the function $\beta=2(1-H)$, where $H$ is the Hurst Parameter.

Assuming $L(k)$ to be a constant, the autocorrelation function and the variance-time function become power functions with decay factor $\beta$ deduced from Eqs. (1) and (4). Using this definition, the long-range dependence of the sampled packet traffic can be determined by calculation of Eqs. (1) and (4), and the Hurst parameter can be estimated.

Figure 5 shows an example of the variance-time curve for the packet traffic bit rate from the Japan Internet exchange (JPIX) to SINET over a $1 \mathrm{~Gb} / \mathrm{s}$ (Gigabit Ethernet) connection. The variance-time curve is calculated using the Variance function with a

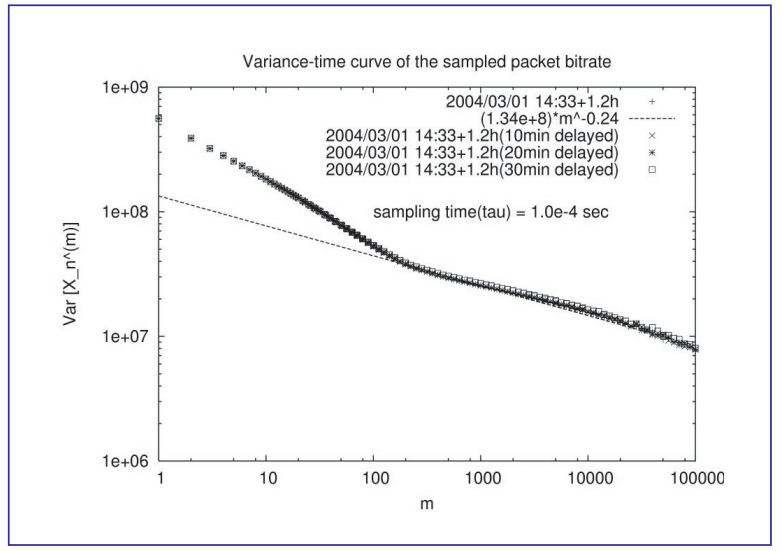

Fig. 5 Variance-time curve for sampled packet bitrate

sampling time of $10^{-4} \mathrm{~s}$ for data extracted over $1.2 \mathrm{~h}$ (14:33-15:45, March 1, 2004). Variance-time curves are also shown for start delays of 10, 20, and $30 \mathrm{~min}$. As all of the curves are very similar, the sampled packet bit rate can be considered to be closely approximate to a weakly stationary process. A Hurst parameter of 0.88 is obtained from the curve of $1.34 \times$ $10^{8} m^{-0.24}$ at large values of $m$. The Hurst parameter can be estimated by a similar process using the Autocorrelation function. The R/S Analysis function, provided as another method to estimate the Hurst parameter, has been described elsewhere [7].

\subsection{Tail probability approximation}

Tail probability is approximated using the FBM traffic model, which has been proposed as a method for analysis of self-similar or long-range dependent traffic $[8,9]$. FBM traffic is defined by

$$
A(t)=\lambda t+\sqrt{\lambda a} Z_{H}(t), \quad t \in \Re,
$$

where $\lambda$ is the arrival rate for packets (packet rate or bit rate), $a$ is the variance of the coefficient for the arrival packet, and $Z_{H}(t)$ is the normalized FBM with zero mean and variance of $\operatorname{Var}\left[Z_{H}(t)\right]=|t|^{2 H}$, where the Hurst parameter satisfies $H \in[0.5,1)$.

FBM traffic defined by Eq. (5) is a cumulative arrival process, and is completely modeled by the three parameters $(\lambda, a, H)$.

The process sampled in the FBM traffic can be considered as follows. If $X_{n}$ is a process sampled in each interval $\tau$ of FBM traffic, and since Eq. (2) can be expressed as $S_{n, m}=A(m \tau)$, the variance-time function (Eq. (4)) becomes

$$
\operatorname{Var}\left[X_{n}^{(m)}\right]=\sigma^{2} m^{-2(1-H)},
$$

where $\sigma^{2}=\lambda a \tau^{2 H}$. 


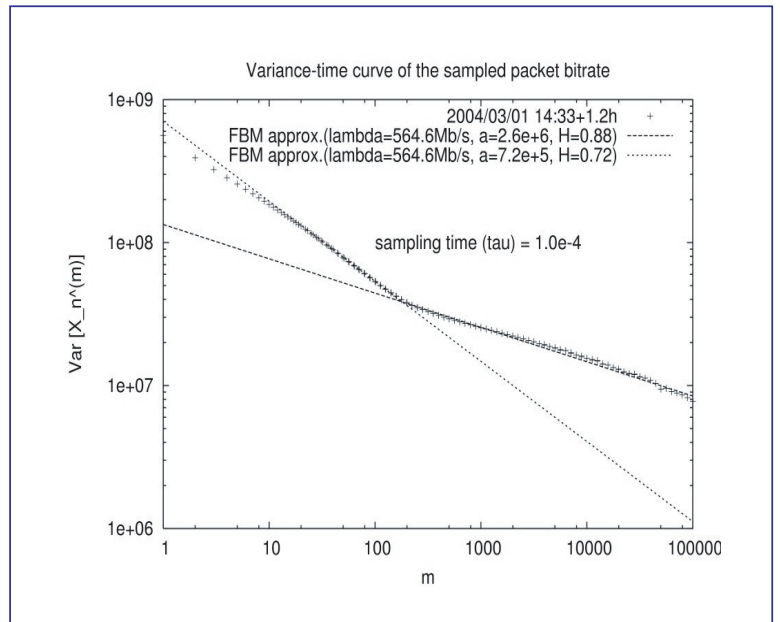

Fig. 6 Variance-time curve approximation by FBM

Comparison of Eq. (4) with Eq. (6) indicates that FBM traffic has long-range dependence. Furthermore, with $r(k)$ as the autocorrelation function for $X_{n}$ and $r^{(m)}(k)$ as the autocorrelation function for $X_{n}^{(m)}$, the following relationship is easily obtained:

$$
r^{(m)}(k)=r(k)=\frac{1}{2}\left\{(k+1)^{2 H}+(k-1)^{2 H}-2 k^{2 H}\right\}(7)
$$

When Eqs. (6) and (7) are satisfied, the model is said to be exactly second-order self-similarity [10]. Thus, FBM traffic has exactly second-order self-similarity and long-range dependence.

The queueing buffer for FBM traffic and service speed $C$ is defined as follows. For queue length $Q$, the tail probability of the queueing buffer is given by [8],[9]

$$
\begin{aligned}
P(Q>x) & \geq \max _{t \geq 0} P(A(t)-C t>x) \\
& =\max _{t \geq 0} \Phi^{\mathrm{c}}\left(\frac{(C-\lambda) t+x}{t^{H} \sqrt{\lambda a}}\right),
\end{aligned}
$$

where $\Phi^{c}(\bullet)$ is the complementary distribution function of the standard Gaussian distribution. The time $t$ satisfying Eq. (8) is given by

$$
t=\frac{H x}{(1-H)(C-\lambda)} \text {. }
$$

Substituting Eq. (9) for Eq. (8) and applying the approximation $\Phi^{c}(u) \approx \exp \left(-u^{2} / 2\right)$ yields the following tail probability approximation:

$$
\mathrm{P}(\mathrm{Q}>x) \approx e^{-\gamma x^{2(1-H)}} ; x \rightarrow \infty,
$$

where $\quad \gamma=\frac{1}{2 \lambda a(1-H)^{2}}\left(\frac{C(1-\rho)(1-H)}{H}\right)^{2 H}$, and

$\rho=\lambda / \mathrm{C}$ is the offered load.

If the observed traffic, that is, the traffic extracted using the Extraction function, can be approximated by FBM traffic, then the tail probability of the queueing buffer with the observed traffic input can be calculated using Eq. (10).

In Fig. 5, the curve $1.34 \times 10^{8} m^{-0.24}$ approximates the variance-time curve of FBM traffic with large values of $m$ with the input parameters $\lambda=564.6 \mathrm{Mb} / \mathrm{s}, \mathrm{a}=2.6$ $\times 10^{6}$, and $H=0.88$. Thus, tail probability can be calculated by Eq. (10) for large time scales (large values of $m$ ). However, for small time scales, tail probability cannot be calculated using these parameters because the variance-time curve deviates from the curve for $1.34 \times 10^{8} \mathrm{~m}^{-0.24}$. Therefore, a variance-time curve at a small time scale is approximated using the FBM curve for the parameter set $\lambda=564.6 \mathrm{Mb} / \mathrm{s}, a=7.2 \times 10^{5}$, and $H=0.72$, as shown in Fig. 6. Using these two parameter sets, tail probability for observed traffic can be calculated over all time scales.

This type of approximation method has been used in previous studies. For example, a parameter set $(\lambda, a$, $H$ ) has been determined for a single case of offered traffic load using the busy period or relaxation time for the queueing buffer, with tail probability calculated using this parameter set [3],[4]. The parameter set has also been determined from the time scale described by Eq. (9) [11],[12]. This latter method allows for simpler calculation of tail probability relative to methods that use the busy period or relaxation time, which have more complex relations. However, the method presented in this study only requires calculation of Eq. (10) using parameter sets that are predefined for certain time-scale ranges.

For a number of FBM parameter sets $n$, defined as $\left(\lambda, a_{1}, H_{1}\right), \ldots,\left(\lambda, a_{n}, H_{n}\right)$, the tail probabilities given by Eq. (10) for each parameter set can be written $P_{1}, \ldots, P_{n}$. For the parameter sets in the downwards convex area of the variance-time curve (see reference [12]), the complete tail probability can thus be approximated by

$$
P(Q>x) \approx \max _{1<i<n}\left\{P_{1}, P_{2}, \ldots, P_{n}\right\}
$$

This approximation estimates tail probability by taking the maximum value of all tail probabilities for all parameter sets. The approximation given by Eq. (11) is shown in Fig. 7 for $\rho=0.85$, along with the results of a simulation using observed traffic for comparison. In this case, the approximation is larger than the simulation, yet by a margin considered acceptable.

This approximation can be calculated using the 
Variance and Tail Prob. Approx. functions.

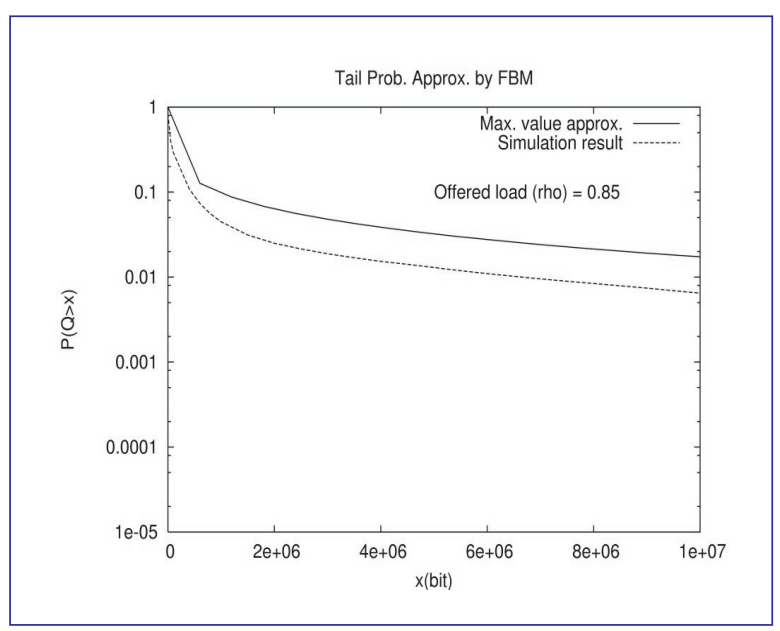

Fig 7 Tail probability approximation by FBM

\subsection{MMPP waiting time approximation}

Since the FBM traffic model is a Gaussian-based approximation, it is imperative that the sampled process can be approximated by a Gaussian process. It has been pointed out that the sampled process is not approximated by a Gaussian process with small time scales [4]. Thus, the FBM traffic model does not apply to small time scales.

Another possible approach consists of a waiting time approximation method using the two-state MMPP traffic model. The two-state MMPP model is a well-known approximation method for analyzing multiplexed voice-packet processes [13]. It should be noted that two-state MMPP has neither self-similarity nor long-range dependence.

Two-state MMPP is completely described by four parameters $\left(\lambda_{1}, \lambda_{2}, r_{1}, r_{2}\right)$ where $r_{1}^{-1}$ and $r_{2}^{-1}$ are the average first- and second-state durations, which obey an exponential distribution, and $\lambda_{1}$ and $\lambda_{2}$ are packet arrival rates obeying a Poisson distribution in the first and the second state. To use two-state MMPP, the four parameters must be determined. In the present case, the four parameters are determined from the variancetime function $\left(\operatorname{Var}\left[X_{n}^{(m)}\right]\right)$ and the third central moment function $\left(\mathrm{T}\left[X_{n}^{(m)}\right] \equiv \mathrm{E}\left[\left\{X_{n}^{(m)}-\mathrm{E}\left[X_{n}^{(m)}\right]\right\}^{3}\right]\right)$ of the sampled packet traffic data. Once the four parameters have been determined, the average waiting time is calculated from the results of MMPP/G/1 analysis using previously published results $[13,14,16]$.

The calculation process is as follows. Let $N(t)$ be the number of arrival packets in $(0, t]$, let $\lambda_{p}$ be the packet arrival rate, and let $\operatorname{Var}[N(t)]$ be the variance of $N(t)$. The IDC is defined by $I(t) \equiv \operatorname{Var}[N(t)] / \mathrm{E}[N(t)]$. Considering the average of $N(t)$, that is, $\mathrm{E}[N(t)]=\lambda_{\mathrm{p}} t$, the IDC of the two-state MMPP is given by [13]

$$
I(t)=I(\infty)-\frac{(I(\infty)-1)^{2}}{2 I^{\prime}(0) t}\left(1-e^{\frac{2 I^{\prime}(0) t}{I(\infty)-1}}\right),
$$

where $I(\infty)=\lim _{t \rightarrow \infty} I(t), I^{\prime}(0)=\lim _{t \rightarrow 0} \mathrm{~d} I(t) / \mathrm{d} t$.

The index of skewness for counts (ISC) is defined as $S(t) \equiv \mathrm{E}\left[\{N(t)-\mathrm{E}[N(t)]\}^{3}\right] / \mathrm{E}[N(t)]$, and is given for the two-state MMPP by

$$
\begin{aligned}
& S(t)=S(\infty)+\{S(\infty)-3 I(\infty)+2\} e^{\frac{-2 I^{\prime}(0) t}{I(\infty)-1}} \\
& -\frac{I(\infty)\{2 S(\infty)-3 I(\infty)+1\}}{2 I^{\prime}(0) t}\left[1-e^{\frac{-2 I^{\prime}(0) t}{I(\infty)-1}}\right],
\end{aligned}
$$

where $S(\infty)=\lim _{t \rightarrow \infty} S(t)$.

Based on results from references [14] and [15], the equations for the four parameters, calculated using IDC and ISC, are as follows:

$$
\begin{aligned}
& r_{1}, r_{2}=D \pm D / \sqrt{1+\lambda_{\mathrm{p}} E}, \\
& \lambda_{1}, \lambda_{2}=\lambda_{\mathrm{p}}+F \pm F \sqrt{1+\lambda_{\mathrm{p}} E},
\end{aligned}
$$

where

$$
\begin{aligned}
& D=I(0) /\{I(\infty)-1\}, \\
& E=I(0) / F^{2},
\end{aligned}
$$

and

$$
F=I^{\prime}(0) \frac{S(\infty)-3 I(\infty)+2}{3\{I(\infty)-1\}^{2}} .
$$

From Eqs. (14) and (15), the four parameters are determined by $\lambda_{p}, I^{\prime}(0), I(\infty)$ and $S(\infty)$, which are derived using the following functions:

$$
\begin{aligned}
& \operatorname{Var}\left[X_{n}^{(m)}\right]=\frac{\lambda_{\mathrm{p}} \tau I(m \tau)}{m}, \\
& \mathrm{~T}\left[X_{n}^{(m)}\right]=\frac{\lambda_{\mathrm{p}} \tau \mathrm{S}(m \tau)}{m^{2}} .
\end{aligned}
$$

First, $\lambda_{p}$ is obtained by calculating the average packet rate directly from the sampled packet data. Next, using the two y-axis values, i.e. $\operatorname{Var}\left[X_{n}^{\left(m_{1}\right)}\right]$ and $\operatorname{Var}\left[X_{n}^{\left(m_{2}\right)}\right]$ on the variance-time curve for the sampled packet data, $I^{\prime}(0)$ and $I(\infty)$ can be obtained from Eqs. (12) and (16). $S(\infty)$ can then be obtained from Eqs. (13) and (17) using one y-axis value $\mathrm{T}\left[X_{n}^{\left(m_{3}\right)}\right]$ on the third central moment curve for the sampled packet data.

An example of variance-time curve approximation with a small time scale is shown in Fig. 8 for the same sampled packet data as that used in Fig. 5. The 
approximation curve was calculated using $I(0)=5.41$ $\times 10^{3}$ and $I(\infty)=2.85$, which were obtained using the values $\operatorname{Var}\left[X_{n}^{\left(m_{1}\right)}\right]=5.09$ and $\operatorname{Var}\left[X_{n}^{\left(m_{2}\right)}\right]=2.97$ for $m_{1}=3$ and $m_{2}=7$ and a packet arrival rate of $\lambda_{p}=86.3 \mathrm{kpps}$. As shown in the figure, the variance-time curve obtained using MMPP provides a good approximation for time scales of $\mathrm{m} \leq 10$. Moreover, given $\left(m_{3}=7, T\left[X_{n}^{\left(m_{3}\right)}\right]=1.12\right), S(\infty)=8.73$ can be obtained from the third central moment of the sampled data.

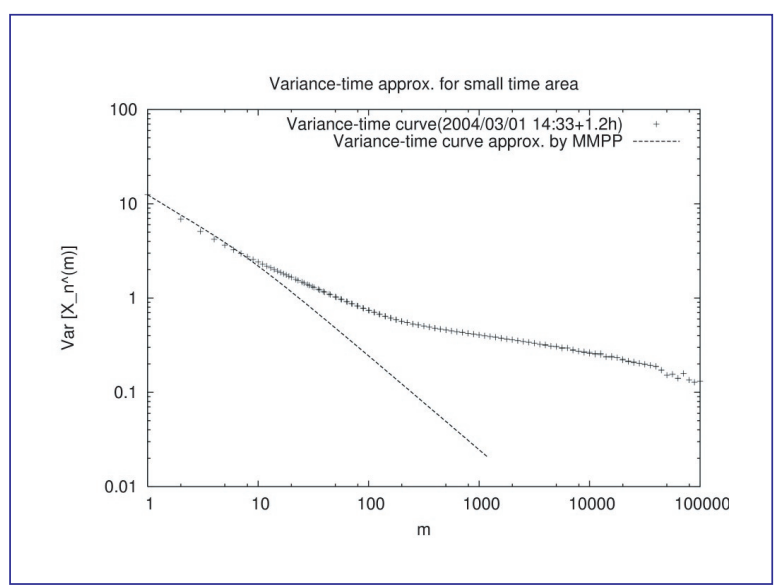

Fig. 8 Variance-time curve approximation by MMPP

For comparison to simulation results for $1.2 \mathrm{~h}$ of extracted data, Figure 9 shows the average waiting time calculated using MMPP/G/1. The MMPP/G/1 calculations were performed using Eqs. (6.21), (6.22) and (6.23) from reference [16]. An exponential distribution was adopted for packet service time because the squared coefficient of variation of the observed service time was approximately 0.7 .

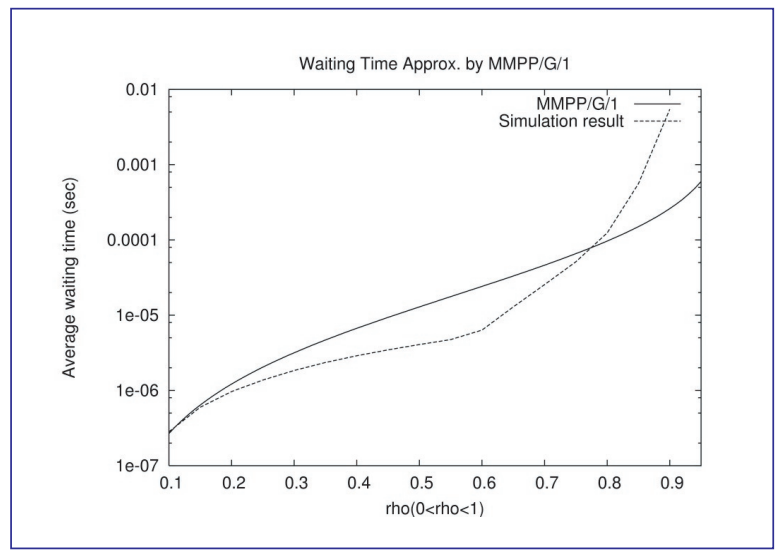

Fig. 9 Waiting time approximation by MMPP/G/1

The average waiting time determined by MMPP/G/1 is larger than the simulation result for $\rho \leq 0.78$, although the approximation is still good. However, the MMPP/G/1 estimate cannot be used for approximation at $\rho \geq 0.78$, as the influence of long-range dependence must be considered in this range.

The proposed approximation methods therefore provide results comparable to those obtained by simulation. However, for analysis of a range of traffic conditions, approximation methods are much simpler than simulations, as simulations must be performed separately for each traffic condition. This is particularly notable in the estimation of tail probability, which can be calculated easily once the FBM parameter sets have been determined. Thus, approximation methods can obtain comprehensive results more efficiently than by simulation. Moreover, exact packet arrival times and packet lengths are required for simulation, which may be difficult to observe at higher transmission speeds. This problem is not as critical for the proposed approximation methods, in which it is sufficient to determine the parameter sets for FMB and MMPP by $\tau$-sampling observation.

\section{Network bandwidth provisioning tool}

The traffic analyzer tools can be used to estimate tail probability and average waiting time for queueing buffers, which also makes these tools applicable to determination of network bandwidth required to achieve a given QoS. Figure 10 shows the process flow for calculation of the network bandwidth. The traffic flow under peak conditions is first estimated from an examination of past traffic, and a peak traffic hour of data is extracted. The extracted traffic is then evaluated to determine whether it can be considered to be a weakly stationary process. If not, another peak traffic hour must be extracted. This check is necessary to ensure an accurate estimation of the Hurst parameter. This can be achieved most easily by plotting several timeshifted variance-time curves on the same graph (e.g., Fig. 5). Following a successful check, the variancetime curve is approximated using FBM variance curves, and the FBM parameters $\left(\lambda, a_{i}, H_{i}\right)$ are determined from the FBM variance-time curves.

An objective offered load $\rho$ is tentatively determined for the network, and tail probability is calculated using this load. If the tail probability is satisfied with respect to a required buffer overflow probability, network bandwidth $C$ is determined by the relation $C$ $=\lambda / \rho$. If the tail probability is not satisfied, a different value of $\rho$ is used and tail probability is recalculated. Note that $\lambda$ can be calculated from the average packet arrival bit rate of the extracted traffic.

WSP checking and FBM variance curve generation are included in the Variance function. Thus, this process can be accomplished by executing the Extraction, 
Variance, and Tail Prob. Approx. functions.

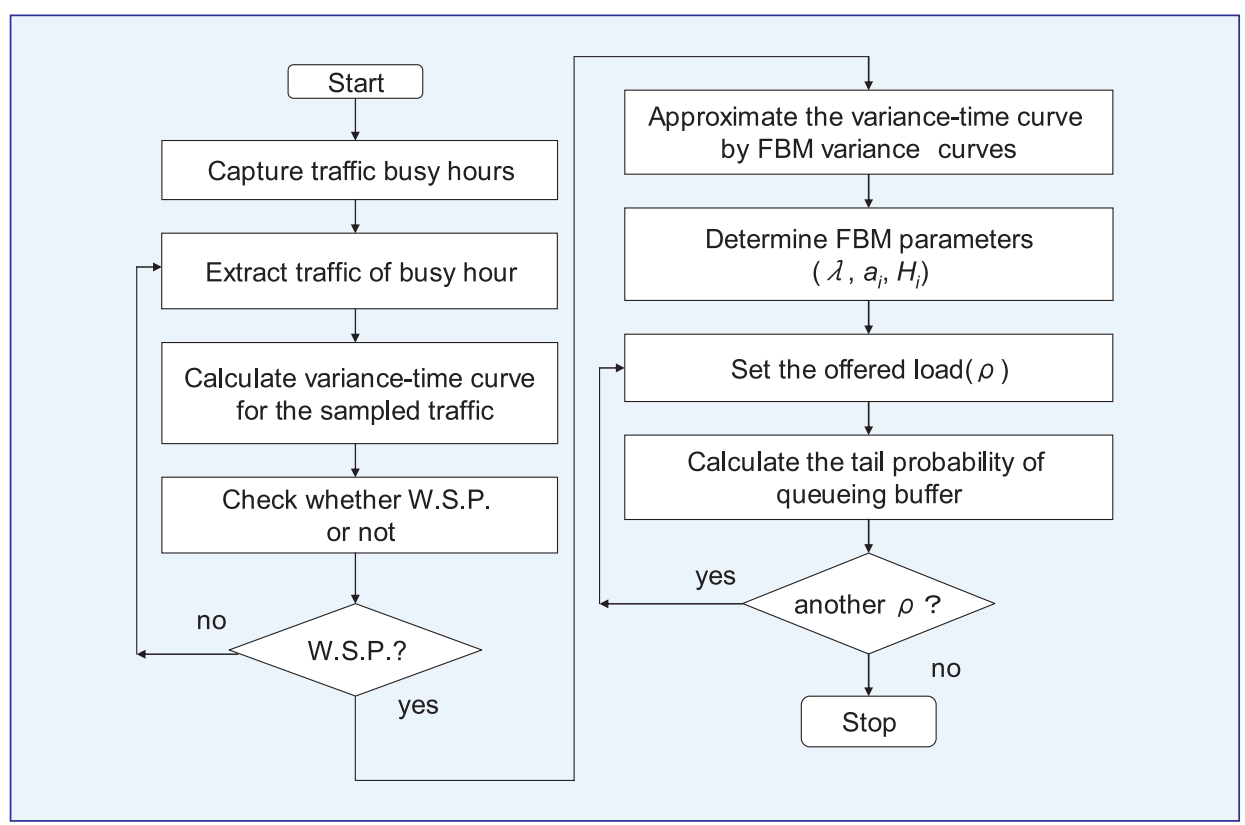

Fig. 10 Processing flow for network bandwidth provision

\section{Conclusion}

The paper presented a range of traffic analysis and network bandwidth provisioning tools developed for network operations in self-similar or long-range dependent traffic environments. In the proposed functions, tail probability is estimated using Fractional Brownian Motion (FBM) tail probability, and average queueing buffer waiting time is determined using the MMPP/G/1 waiting time approximation method. The estimations by these two methods are consistent with the results of simulations based on observed traffic data. These tools can also be used to determine network bandwidth.

\section{References}

[1] W. E. Leland, M. S. Taqqu, W. Willinger, and D. V. Wilson, "On the self-similar nature of Ethernet traffic (extended version)," IEEE/ACM Trans. Networking, vol. 2 pp. 1-15, 1994.

[2] V. Paxson and S. Floyd, "Wide area traffic: The failure of Poisson modeling," IEEE/ACM Trans. Networking, vol. 3, no. 3, pp. 226-244, 1995.

[3] S. Abe, T. Fujino, Y. Ji, J. Matsukata, and S. Asano, "Autocorrelative traffic analysis on an academic information network," Internet Conference 2001, pp. 151-158, 2001.
[4] S. Abe, T. Fujino, Y. Ji, J. Matsukata, and S. Asano, "Long-range dependent traffic analysis and approximation method of queue-length probability for an academic information network," Trans. IEICE, vol. J86-B, no. 12, pp. 2487-2500, 2003. (in Japanese)

[5] S. Abe, T. Fujino, Y. Ji, J. Matsukata, and S. Asano, "Performance evaluation method by using observed traffic data for an academic information network," Tech. Rep. IEICE, IN2003-139, pp. 31-36, 2003. (in Japanese)

[6] http://www.tcpdump.org/

[7] J. Beran, "Statistics for long-memory processes," Chapman\&Hall/CRC, 1994.

[8] I. Norros, "A storage model with self-similar input," Queueing Syst., vol. 16, pp. 387-396, 1994.

[9] I. Norros, "On the use of fractional brownian motion in the theory of connectionless network," IEEE J. Select. Areas Commun., vol. 13, no. 6, pp. 953-962, 1995.

[10] T. Ozawa, "Traffic models with long-range dependence and self-similarity," Syst. Contr. Inform., vol. 43, no. 3, pp. 117-122, 1999. (in Japanese)

[11] Y. Ji, T. Fujino, S. Abe, J. Matsukata, and S. Asano, "On the impact of time scales on tail behavior of longrange dependent Internet traffic," Proc. 11th IEEE Int. Conf. on Networks, pp. 13-18, 2003.

[12] Y. Ji, T. Fujino, S. Abe, J. Matsukata, and S. Asano, "Performance analysis of long-range 9 dependent Internet traffic according to relevant time scales," 
Trans. Inform. Process. Soc., vol. 45, no. 5, pp. 1399-1408, 2004. (in Japanese)

[13] H. Heffes and D. M. Lucantoni, "A Markov modulated characterization of packetized voice and data traffic and related statistical multiplexer performance," IEEE J. Select. Areas Commun., vol. 4, no. 6, pp. 856-868, 1986.

[14] T. Okuda, H. Akimaru, and M. Sakai, "A simplified performance evaluation for packetized voice systems," Trans. IEICE, vol. E73, no. 6, pp. 936-941, 1990.

[15] S. Abe, "Performance evaluation by renewal process approximation for a queueing system with multiplexed burst packet inputs," Electron. Commun. Jpn. 1, vol. 80, no. 7, 1997.

[16] H. Akimaru and K. Kawashima, "Information and communication traffic - fundamentals and application-," The Telecommunications Association, 1990. (in Japanese)

[17] D. R. Cox and P. A. W. Lewis, "The statistical analysis of series of events," Chapman and Hall, 1966.

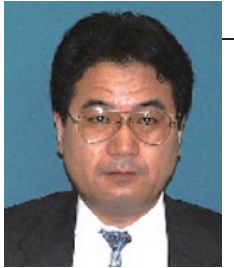

Shunji ABE

Shunji Abe received B.E. and M.E. degrees from Toyohashi University of Technology, Japan, in 1980 and 1982, respectively. He received a Ph.D. from the University of Tokyo in 1996. In 1982 he joined Fujitsu Laboratories Ltd., where he engaged in research on broadband circuit switching system, ATM switching system, ATM traffic control, and network performance evaluation. He worked at the National Center for Science Information Systems, Japan (NACSIS) from 1995 to 1999. Since 2000 he has worked at the National Institute of Informatics of Japan as an associate professor. He is also an associate professor of the Graduate University for Advanced Studies (SOKENDAI). He is currently interested in the Internet traffic analysis, network performance evaluation, optical switching system architecture, and mobile IP system architecture. He is a member of IEEE.

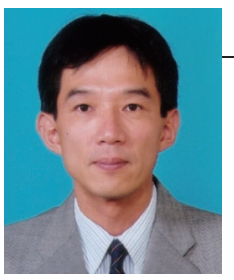

Toru HASEGAWA

Toru Hasegawa received the B.E., the M.E. and Dr. Informatics degrees in information engineering from Kyoto University, Japan, in 1982, 1984 and 2000, respectively. Since joining KDD in 1984, he has been working in the field of formal description technique (FDT) of communication protocols. From 1990 to 1991, he was a visiting researcher at Columbia University. His current interests are mobile computing and high-speed protocol. He is currently an executive director of KDDI R\&D Laboratories Inc. He is also a guest professor at National Institute of Informatics. He received The Meritorious Award on Radio of ARIB in 2003.

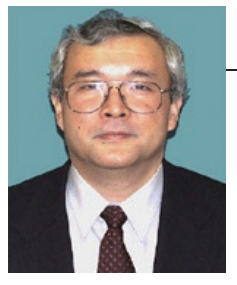

Shoichiro ASANO

Shoichiro Asano had graduated at Electronic Engineering, Faculty of Engineering, University of Tokyo, in 1970. He received $\mathrm{ME}$ and DE, both from University of Tokyo, in 1972 and 1975 respectively. Dr. Asano is a Director and Professor, Infrastructure Systems Research Division, National Institute of Informatics (NII) and a Professor, Graduate School of Information Science and Technology, University of Tokyo. His current researches are mainly focused on Optical Network Architecture and development of SuperSINET in Japan. 